\title{
ТВОРЧЕСКОЕ МЫШЛЕНИЕ В КОНФЛИКТНОЙ СИТУАЦИИ
}

Глубинные трансформационные процессы Армянского общества затронули важнейшие сферы жизнедеятельности людей и глубоко проникли в общественные и межличностные отношения. Неблагоприятная социальная среда, отрицательно влияя на психическое состояние человека, приводит к снижению его работоспособности, обусловливает рост напряженности, конфликтности, агрессивности, и все это, так или иначе, влияет на возникновение межличностных конфликтов. В связи с этим умение оптимально вести себя в конфликтных ситуациях предполагает как понимание причин и механизмов возникновения конфликтов, так и анализ конфликтных ситуаций и креативного подхода в их решении. Использование творческого мышления позволяет выработать правильный способ поведения людей в конфликтных ситуациях, находить оптимальные пути выхода из конфликта.

В настоящее время проблема творческого мышления и конфликтности личности набирает свою значимость и требует подробного теоретического и прикладного исследования.

В современной психологии конфликты рассматривались в следующих направлениях:

1. следствие действующих на человека разнонаправленных сил (мотивов),

2. результат определенной ситуации конкурентного или кооперативного взаимодействия людей,

3. сложная многоуровневая система взаимозависимых компонентов, имеющий неоднозначные функции,

4. состояние более или менее длительной дезинтеграции личности, выражающееся В обострении или в возникновении новых противоречий.
Большой вклад в разработку теории конфликтов внесли также Л.А.Петровская, Ф.М.Бородкин, Н.М.Коряк, Н.В.Гришина, С.Геворкян, А.И.Донцов, Т.А.Полозова, и др. Проблеме мышления и, в частности творческого мышления, посвящено большое количество исследований, которые рассматривают различные особенности процесса мышления в ходе решения задач и проблемных ситуаций (А.Н.Леонтьев, С.Л.Рубинштейн, О.К.Тихомиров, А.М.Матюшкин, Д.Б.Богоявленская, Я.А.Пономарев, В.Н.Дружинин, К.Дункер, М.Вертгеймер, К.Восканян, С.Медник и др.). Однако на сегодняшний день недостаточно разработанной остается проблема особенностей мышления в конфликтных ситуациях.

Построение строгих теорий протекания внутренних мыслительных процессов в системе взаимодействия людей в конфликтных ситуациях наталкивается на большие трудности, так как речь идет о взаимодействии объективных факторов реальной ситуации и субъективных факторов внутриличностного характера, связанных с особенностями познавательных процессов, опосредованных индивидуальными особенностями субъектов взаимодействия, в том числе и столкновении нескольких субъективных реальностей. Сама конфликтная ситуация в данном случае является источником мыслительной и творческой деятельности субъекта, звеном непосредственно связывающим мышление и деятельность личности. Мышление в конфликтной ситуации, на наш взгляд, характеризуется особенностями творческого мышления, необходимыми для успешного разрешения конфликтной ситуации и предотвращения собственно конфликтного взаимодействия. 
С точки зрения межличностного аспекта конфликта, включающего познание другого человека и подготовку воздействия на него, ранее нами обсуждался вопрос искажения восприятия в межличностном познании, и его роли в развитии агрессии. (Тевосян М.В. 2001) Именно оценка и интерпретация намерений окружающих и атрибуции причин привлекла наибольшее внимание исследователей, что подчеркивает особую значимость этого этапа в общей цепи развития всего процесса деятельности личности в конфликте. Однако не только люди, но и ситуации являются объектом познания в межличностном взаимодействии, а более полная модель выглядит как «люди в ситуациях». То есть ситуация является тем контекстом, где разворачиваются события и действуют люди. В данной работе мы считаем необходимым проанализировать поэтапное развитие мышления и выявить роль творческого мышления в конфликтных ситуациях.

Учитывая особенности рассматриваемой проблемы необходимо конкретизировать понятия «внешних и внутренних условий» мыслительной деятельности. Среди характеристик внешних условий можно выделить, например, число «степеней свободы» выбора способов преобразования условий, число правильных путей к цели, зависимость или независимость преобразования условий задачи от субъекта, наличие или отсутствие конфликта ценностных характеристик преобразований ситуации, а далее анализировать «вклад» этих конкретных характеристик в детерминацию мыслительного процесса. Таким образом, анализ структуры самих задач и их влияния на ход мыслительного процесса составляет один из путей «расшифровки» «внешних условий» мышления. Необходимо только дифференцировать формальную и неформальную (т.е. смысловую и ценностную) структуру задачи. Кроме самой задачи к «внешним условиям» относятся общая об- становка деятельности и вся система воздействий, которая может возникать в ситуации межличностного общения.

Каждый раз в течении жизни, оказываясь в новой, проблемной ситуации и, приступая к разрешению определенного противоречия, субъект представляет собой совокупность внутренних условий, которые опосредуют все внешние воздействия, в том числе и требования деятельности им выполняемой. В качестве внутренних условий выступают как предыдущие внешние воздействия, интериоризированные, и выступающие на данный момент в качестве внутренних, так и наследственные и врожденные особенности субъекта, т.е. изначально внутренние. В деятельности, направленной на разрешение ситуации происходит обогащение, обновление и соответственно развитие способностей личности.

Если в рамках психологического содержания понятия «внутренние условия» мышления С.Л.Рубинштейн обсуждает «процессы анализирования, синтезирования, обобщения», то О.К.Тихомиров считает необходимым добавить возникновение и сложную динамику эмоциональных оценок, невербализованных смыслов, предвосхищений, «предгипотез», изменение установок в ходе решения задачи, возникновение и удовлетворение познавательных потребностей (Рубинштейн 1998).

Обсуждая процесс решения задачи разными способами, К.В.Восканян выделяет два основных этапа развития мышления (Восканян 2001). На первом этапе посредством актуализации имеющихся знаний субъект приписывает объекту определенные характеристики. Этот этап характеризируется автором как точка отсчета мыслительной деятельности. На втором этапе происходит переосмысление предположений выдвинутых на первом этапе. Основной функцией этого этапа является вычленение новых свойств объекта, посредством анализа, с открытием нового, объективного со- 
держания познаваемого объекта. Предположения, сделанные на данном этапе, носят прогностический характер. Выявление нового содержания приводит к изменению проблемной ситуации, где задача превращается в процесс, посредством осмысления проблемной ситуации. С переходом из одной ситуации в другую, происходит поэтапное перестраивание мышления субъекта.

Раскрытие нового содержания и перестройка мышления субъекта, а следовательно, и перестраивание самой ситуации, на наш взгляд происходит посредством творческого мышления. Творческий акт заключается в высвобождении мышления от тех стереотипов, которые сделали ситуацию неразрешимой.

Если согласиться с тем, что творческое мышление является высшей формой мышления, то на основе наиболее значимых теорий мышления можно выделить наиболее существенные свойства творческого мышления как психологического феномена.

При характеристике творческого процесса обычно подчеркивается способность человека видеть предмет с новой стороны и наличие, по крайней мере, двух моментов: непроизвольного (хотя и подготовленного) генерирования новых образов и идей и контроль, оценка этих образов и идей в соответствии с конкретными задачами (интуитивный и логический компоненты).

Обратившись к теории мышления А.В.Брушлинского, следует отметить, что творческое мышление характеризуется двумя основными специфическими свойствами: особой ролью субъекта познания и вероятностным прогнозированием как основной функцией (Брушлинский 1979). Только при единстве этих двух условий можно говорить о наличии творческого мышления в структуре когнитивных функций субъекта.

При этом процесс мышления связан с созданием модели проблемной ситуации из на- бора структур и схем зданий, хранящихся в долговременной памяти. В случае относительно простой задачи, субъект, обладая хорошо структурированными знаниями, достаточно легко создает адекватную модель. Однако отсутствие таких структур в долговременной памяти превращает задачу в творческую. Обсуждая проблемы творчества, Пономарев приходит к выводу, что процесс творчества связан с особенностями стоящих перед человеком задач.

Как отмечает КашаповМ.М «любая подлинная деятельность по природе своей конфликтна». (Кашапо 2003: 183). Даже проявление инициативы может быть рассмотрено как противоречие общепринятому. Творчество означает, прежде всего, нарушение догм.

Сама конфликтная ситуация представляет собой некоторый процессуальный комплекс, образованный из процессов переорганизации деятельности в связи с новыми обстоятельствами, оформления новых предметов деятельности для их преобразования, мобилизации ресурсов для овладения ситуацией.

Одна из важнейших процессуальных характеристик конфликта - это привлечение новых ресурсов.

Неслучайно Хасан Б.И. определяет конфликт как стимул, требующий творческой реакции, который приводит к формированию новых черт личности. Именно в этом заключается его развивающая функция (Хасан 2001: 229).

Если следовать в русле размышлений о развитии Б.Д.Эльконина, то построение нового средства, преобразующего ситуацию, и открытие для себя именно этой способности - к «построению действия» - есть то условие, при котором в конфликте, в его разрешении совершается творческий акт, а значит, и развитие (Эльконина 1994).

Ситуативная характеристика конфликта определяется лежащим в его основании противоречием, составляющим проблемное поле 
конфликта. В качестве момента, дополняющего понимание проблемной ситуации как феномена можно привести замечание М.М.Кашапова: «Проблемность ситуация приобретает при обнаружении в ней противоречий и порождает процесс мышления, направленный на «снятие» противоречий» ( Кашапова 2001: 195).

Под содержанием проблемной ситуации мы понимаем следующие ее особенности: характер этой проблемной ситуаций (что собственно является сутью проблемности) и направленность искомого решения (в каком направлении происходит его поиск). В современной когнитивной психологии соответственно выделяются два этапа в процессе мышления: этап создания модели проблемной ситуации и этап оперирования с этой моделью, понимаемый как поиск в проблемном пространстве.

Когнитивная теория Дж.Брунера акцентирует внимание на двух принципиальных информационных стратегиях мышления: 1) парциальном сканировании (симультанном-беспорядочном и сукцессивном - последовательном) и 2) «фокусировке» (Брунер 1977).

Природе творческого мышления, несомненно, соответствует стратегия «фокусировки», т.к. именно выделение основной сути, ядра проблемной ситуации является первичной задачей процесса творческого поиска. Вероятно, фокусировка в творческом мышлении может быть как консервативной, связанной с оценкой вариантов в зависимости от степени их объективной вероятности, так и азартной, основанной на интуитивном поиске и проверке, часто связанными с риском неудачи или ошибки.

В данном контексте можно провести параллели между сложной шахматной позицией, включающей очень большое число отношений между фигурами и конфликтной ситуацией, включающей аналогичную сложную структуру взаимоотношений. На первом этапе, создания представления о задаче, построение модели ситуации, субъект деятельности по необходимости отбирает только некоторые из этих отношений, наиболее существенные с его точки зрения. На основе этих отношений и строится «проблемное пространство».

Модель проблемной ситуации возникает не на пустом месте: в ее создании участвуют структуры и схемы знания, находящиеся в долговременной памяти и посредством мышления ситуации дается определение. При этом, если субъективная картина ситуации расходится с имеющимися у человека представлениями о данном классе ситуаций, возможна либо корректировка общей модели, либо «подгонка» ситуационной модели под общую, отвечающая известному явлению стереотипизации. Согласно представлениям У.Клара и его коллег о конфликте как когнитивной схеме: если ситуация определяется как конфликтная, будут выбираться доказательства, поддерживающие эту схему (Klar, Bar-Tal, Kruglanski 1988). Следовательно, можно сделать вывод о том, что, скорее всего имеет место «схватывание» ситуации в целом, тогда как ее опознание «по деталям» вызывает затруднение. Сталкиваясь с затрудняющей его ситуацией, человек старается в первую очередь отнести ее к определенному типу, о котором он имеет более-менее ясные представления. Стремление человека к «определению ситуации» обусловлено желанием снизить дискомфорт от чувства неопределенности. По Сикурелю, «интерпретация - это постоянный процесс, где структурное сходство контекста с другими известными событиями («чувство социальной структуры») активизирует различные мыслительные схемы и типизации. Такой сплав с намерениями индивида конструирует новую действительность и новое знание» (Бекк-Виклунд 1992: 98).

В случае несоответствия имеющихся знаний и опыта, часто возникает осознание безысходности в конфликтной ситуации, что фруст- 
рирует индивида, ограничивает свободу выбора, сводя количество альтернатив до минимумa.

Однако одно дело думать о чем-то, другое дело - придумывать. В процессе обдумывания выявляются новые отношения, те, которые раньше не воспринимались как существенные. Для объяснения того, что происходит при размышлении, можно воспользоваться термином С.Л.Рубинштейна «анализ через синтез»: выявление новых отношений фигур (анализ) происходит в результате переосмысления позиции и постановки новых целей (синтез) (Рубинштейна 1998).

А.В.Брушлинский пишет: «...Человек ищет и находит решение мыслительной задачи не по принципу выбора из альтернатив, а на основе строго определенного, непрерывного, но не равномерно формирующегося прогнозирования искомого» (Брушлинский 1979: 159).

В процессе создания нового многие авторы выделяют вопрос о роли сознательного и бессознательного, иначе говоря, о соотношении интуиции и логики в творческом процессе решения проблемной ситуации. Выделяя два вида опыта субъекта: интуитивный и логический, Я.А.Пономарев считает, что интуитивный опыт, обладая весьма своеобразными свойствами, может быть назван бессознательным по двум причинам: во-первых, он образуется помимо воли субъекта и вне поля его внимания; во-вторых, он не может быть произвольно актуализирован субъектом и проявляется только в действии. Логический опыт, напротив, осознан и может быть применен при возникновении соответствующей задачи.

При решении задачи, которая не является принципиально новой для субъекта, решающий, функционируя на логическом уровне, актуализирует нужные знания и создает адекватную модель. При столкновении с необычной задачей логические знания субъекта оказываются недостаточными. Тогда функциони- рование психического механизма, по выражению Я.А.Пономарева, “спускается» на более интуитивные уровни (Пономарева 1988). В сфере интуиции опыт менее структурирован, но более богат, поэтому там субъекту иногда удается найти ключ к решению задачи. Если принцип решения на интуитивном уровне найден, субъекту нужно еще оформить его в виде хорошо структурированной модели, перевести на логический уровень.

Я.А.Понамарев выделяет четыре фазы творческого процесса :

1. Сознательная работа. субъект безрезультатно использует логические методы решения. Особое деятельное состояние как предпосылка интуитивного проблеска новой идеи.

2. Бессознательная работа. Созревание, решающий оставляет сознательные попытки, однако взамен включается интуитивный уровень мышления.

3. Переход бессознательного в сознание. Этап вдохновения. В результате бессознательной работы в сферу сознания поступает идея решения. Первоначально в виде гипотезы, в виде принципа или замысла.

4. Сознательная работа. Развитие идеи, окончательное оформление идеи.

В основу выделения фаз Я.А.Пономарев закладывает: - переход от сознательного поиска к интуитивному решению; - эволюция интуитивного решения в логически завершенное.

Вопрос особенностей интуиции и логики и их соотношения в процессе принятия творческого решения требует более детального рассмотрения. Современные ученые определяют логику как метод познания и доказательства, основанный на применении таких операций, как индукция, дедукция и аналогия (Новиков 2000).

В связи с тем, что разные идеи вытекают из своих исходных посылок с разной степенью 
вероятности, логику подразделяют на строгую и нестрогую. В строгой логике исходные посылки однозначно определяют конечный вывод, а также существует гарантия истинности заключений в случае истинности исходных посылок. В нестрогой же, то есть вероятностной логике конечный вывод не вытекает однозначно из исходных посылок и нет гарантии достоверности заключений в случае достоверности исходной информации.

Для нестрогой логики характерно стремление путем обобщения уже имеющихся знаний выйти за его пределы, сформулировать новые положения, истинность которых еще не доказана. В данном случае вывод по неполной индукции и аналогии носят вероятностный характер, так как представляет собой своего рода скачок, переход от известного к неизвестному. «Если строгая логика, такая, как дедуктивное мышление, легко поддается аксиоматизации и формализации, то нестрогая логика не может служить надежным алгоритмом, введение которого в компьютер позволило бы автоматизировать процесс творческого мышления" (Новиков 2000: 114).

Интуиция представляет собой прямое, непосредственное усмотрение истины без обоснования логическими доказательствами и аргументами, характеризуется внезапностью, неосознаваемостью, способностью детерминировать разрывы и пробелы в поэтапном процессе переработки наличных данных, порождать свертывание и кристаллизацию ментального опыта, не поддается формализации и аксиоматизации. В интуитивной теории процесс возникновения идей описывается как процесс, не обусловленный исходными посылками, не имеющий той базовой информации, которая привела к рождению оригинального творческого продукта.

Поскольку критерием творческого акта, согласно Я.А.Пономареву, является уровневый переход от низших к высшим структурным уровням, что приводит к возникновению нового способа взаимодействия субъекта с объектом и возникновению нового знания. Тем самым творческий продукт предполагает включение интуиции и, по мнению автора, не может быть получен на основе логического вывода. Однако мы считаем, что интуиция и логика в данном контексте являются не взаимоисключающими, а взаимодополняющими аспектами развития мышления в целом и его творческих составляющих в частности.

На основании анализа интеллектуальной деятельности выдающихся ученых и решения творческих задач обычными людьми Н.Б.Новиков приходит к выводу, что «новые идеи и решения возникают в результате совместного участия логического и интуитивного мышления человека, благодаря взаимному функционированию логики и интуиции в конкретных проблемных ситуациях» (Новиков 2000: 115). Причем, речь идет именно о вероятностной логике как частном проявлении логических аспектов. Автор так же подчеркивает, что для интуиции характерно не отсутствие посылок, а их наличие в виде личностных убеждений, предпочтений и верований, объединяющихся в понятии интенционального опыта.

Образование новизны за счет комбинаций элементов можно определить термином инсайта. Даже длительный и интенсивный процесс творческого мышления исследователя может прерваться из-за недостатка в памяти или в текущей информации какого-то звена, необходимого для завершения разумной комбинации. А спустя некоторое время, получив извне, может быть случайное, но необходимое или в чем-то близкое к нему воздействие-подсказку, в мозге исследователя происходит неожиданное завершение или смена комбинации. Теперь уже на сознательном уровне, после уточнения деталей, формируется продукт мыслительного процесса. 
Определяя дивергентное мышление как «тип мышления, идущего в различных направлениях», Дж.Гилфорд считал операцию дивергенции, наряду с операциями преобразования и импликации, основой креативности как общей творческой способности (Гилфорд 1965). Дивергентное мышление предполагает, что на один и тот же вопрос может быть дано множество разнообразных, но одинаково равноправных ответов, в отличие от конвергентного мышления, которое ориентировано на единственно верное решение, с точки зрения предыдущего опыта и усвоенных знаний. Следовательно, чем больше вариантов решения кон $\phi-$ ликтной ситуации человек видит и осознает, тем выше вероятность реализации оптимального способа разрешения. Можно заключить, что конвергентное мышление на начальном этапе построения задачи, скорее всего приведет к тупиковой ситуации, создавая основу для ощущения неразрешимости проблемы. Однако, оно так или иначе необходимо для построения предварительной модели ситуации, на основании имеющегося опыта. Однако дальнейшее развитие мышления по необходимости предполагает нахождение новых возможных вариантов «видения» проблемы и следовательно вероятных способов разрешения. Далее все же необходимо из имеющихся вариантов выбрать одно, самое оптимальное, самое целесообразное решение, что снова активизирует мыслительную операцию конвергенции.

Согласно концепции А.Медника лежащей в основе разработанного им теста RAT (тест отдаленных ассоциаций), процесс дивергентного мышления идет посредством мыслительного поиска в разных направлениях семантического пространства, как бы отталкиваясь от содержания проблемы (Mednich 1969). Дивергентное мышление рассматривается как боковое, периферическое мышление, мышление «около проблемы». Конвергентное мышление увязывает все элементы семантического пространст- ва, относящиеся к проблеме, воедино, находит единственно верную композицию этих элементов. Медник полагает, что в творческом процессе присутствует как конвергентная, так и дивергентная составляющие. По мнению Медника, синтез элементов может быть нетворческим и стереотипным, следовательно, суть творчества, не в особенности операции, а в способности преодолевать стереотипы на конечном этапе мыслительного синтеза.

Вышеописанные фазы и отдельные составляющие процесса мышления, позволяют составить описание более обобщенной модели поэтапного развития мышления в процессе разрешения противоречия в конфликтной ситуации, и соответственно представить процессуальную характеристику мышления в конфликте. Оставаясь в рамках двух основных фаз - построения модели проблемной ситуации и поиска решения в проблемном поле конфликтной ситуации, мы считаем, что первую фазу можно разделить на два основных этапа, а вторую - на три.

І фаза - На первом этапе творческое мышление проявляется в способности увидеть, вычленить само противоречие, суть проблемы в пространстве конфликтной ситуации. Выделение существенных с точки зрения решения проблемы признаков, позволяет построить более адекватную картину ситуации, потенциально содержащую так же возможности реконструкции и нахождения способов разрешения. На данном этапе осуществляются как процессы анализа, так и синтеза. Однако, исходя из особенностей проблемного поля конфликтной ситуации, которая характеризуется новизной, неопределенностью, необходимостью привлечения новых ресурсов, прежний опыт может оказаться недостаточным и первичная модель проблемного поля не будет удовлетворительной для решения проблемы.

На втором этапе преодоление состояния неразрешимости противоречия происходит 
посредством более разностороннего анализа. С помощью вероятностной логики и, особенно, интуиции происходит выявление новых, до этого не подмеченных свойств ситуации, взаимосвязей познаваемых объектов, которые появляются в качестве инсайта. В результате творческих процессов происходит построение новой модели, раскрывающей потенциальные возможности решений.

II фаза - На первом этапе происходит генерация различных возможных вариантов решений, на основании более широкого охвата проблемного поля конфликтной ситуации. Творческое мышление проявляется в таких характеристиках как беглость, гибкость, сложность, оригинальность.

На втором этапе надо определиться с вариантом решения, творческая составляющая этого выбора проявляется в выборе из возможных альтернатив наиболее нестандартного способа, однако этот способ должен так же соответствовать критерию практичности, «применимости» в данной ситуации, что само собой соответствует требованиям третьего этапа данной фазы.

На третьем этапе происходит практическая проверка, реализация замысла решения в конкретной ситуации.

Таким образом, на основании анализа различных подходов, мы пришли к выводу об

\section{ЦИТИРУЕМАЯ И УПОМИНАЕМАЯ ЛИТЕРАТУРА}

1. กuluu\{ju\{, Ч.Ч. (2001). Eưuphphl lu untuuluwa nanhuiapugnuduteph ntenn un-

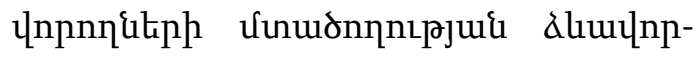

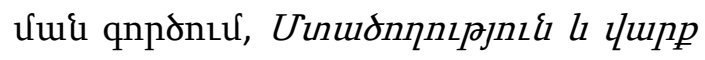

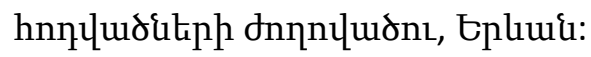

2. Бекк-Виклунд, М. (1992). Феноменология: мир жизни и обыденного знания // П.Монсон. Современная западная социология: теории, традиции, перспективы. Спб. особой роли и месте творческого мышления в конфликтной ситуации. Причем творческое мышление опосредует динамику поиска и нахождения решения на всех этапах развития процесса. Если главная функция интеллекта заключается в построении адекватных репрезентаций происходящего, то отличительной характеристикой творческого мышления определенным образом является способность по-иному видеть, понимать и интерпретировать окружающую действительность. Тип и способ репрезентации происходящих событий выступает в качестве одного из базовых феноменов интеллектуальной жизни человека и его опыта в целом. Однако реорганизация и переструктурирование репрезентативной модели в процессе разрешения конфликтной ситуации, выступающей в качестве стимула для формирования нового опыта, есть функция творческих процессов мышления. Следовательно, мы полагаем, что всесторонний анализ творческих особенностей мышления, позволит подходить конструктивно к преодолению конфликтной ситуации, посредством разрешения лежащей в основе проблемы-противоречия, и эффективно предвосхищать эскалацию конфликтных отношений.

3. Брунер, Дж. (1977). Психология познания. За пределами непосредственной информации. М.

4. Брушлинский, А.В. (1979). Мышление и прогнозирование. М.

5. Бэрон, Р., Ричардсон, Д. (2001). Агрессия, СПб.

6. Гилфорд, Д. (1965).Три стороны интеллекта // Психология мышления / Ред. А.М.Матюшкин. М.

7. Кашапов, М.М. (2003). Теория и практика решения конфликтных ситуаций. Учеб- 
ное пособие /Под научн ред проф А.В.Карпова, М, Ярославль.

8. Кашапов, М.М. (2001). Методологическая основа профессионального педагогического мышления //Психология субъекта профессиональной деятельности. / Под ред. А.В.Брушлинского, А.В.Карпова. М. - Ярославль.

9. Новиков, Н.Б. (2000). Соотношение интуиции и логики в процессе порождения новых научных идей. // Интеллект и креативность в ситуациях межличностного взаимодействия, сборник научных статей, Российская академия наук Институт психологии, Москва.

10. Пономарев, Я.А. (1988). Психология творчества // Тенденции развития психологической науки. М.

11. Рубинштейн, С.Л. (1998). Основы общей психологии. СПб.с

12. Тевосян, М.В. (2001). Восприятие и мышление в конфликтной ситуации. Влияние когнитивных процессов на развитие аг-

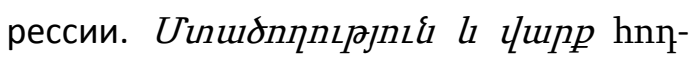

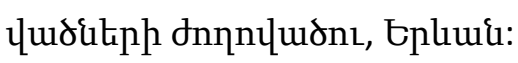

13. Хасан, Б.И., Сергоманов П.А. (2001). Разрешение конфликтов и ведение переговоров. Учебное пособие, Красноярск Москва.

14. Эльконин, Б.Д. (1994). Введение в психологию развития. М.

15. Klar, Y.; Bar-Tal, D.; Kruglanski, A. (1988). Conflictas a Cognitive Schema: Toward a Social Cognitive Analysis of Conflict and Conflict Termination // The Social Psychology of Intergroup Conflict. Theory, Research and Applications / Ed. By W.Stroebe a. o. Berlin.

16. Mednich, S.A. (1969). The associative basis of the creative process // Psychol. - Rewiew. - № 2.

ТЕВОСЯН МАРИЯ

\section{ТВОРЧЕСКОЕ МЫШЛЕНИЕ В КОНФЛИКТНОЙ СИТУАЦИИ}

\section{PEЗЮME}

В статье обсуждается роль творческого мышления в конфликтной ситуации. В связи с проблемой исследования конкретизируются понятия «внешних и внутренних» условий мыслительной деятельности, где конфликтная ситуация выступает в качестве связующего звена мышления и деятельности. На примере моделей, предложенных разными авторами, всесторонне анализируется процесс мышления в ходе решения проблемных ситуаций. В частности обсуждается проявление различных особенностей и процессов мышления, таких как логика, интуиция, конвергенция, диверген- ция, на разных этапах развития процесса мышления. Представляется обобщенная модель мышления в процессе поиска решения в проблемном поле конфликтной ситуации, где отдельно рассматриваются функции интеллекта и творческого мышления.

Ключевые понятия: конфликтная ситуация, проблемная ситуация, модель проблемной ситуации, интуиция, логика, творческое мышление, «внешние и внутренние условия» мыслительной деятельности, конвергентное и дивергентное мышление. 


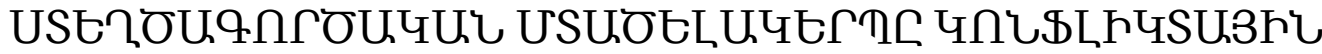

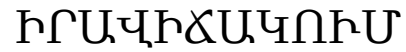

\section{UUФกФกЋU}

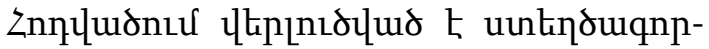

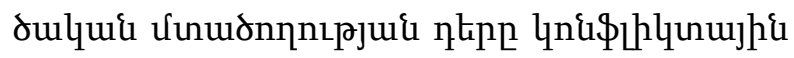

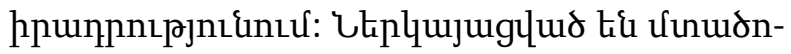

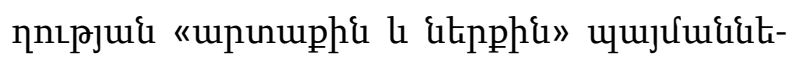

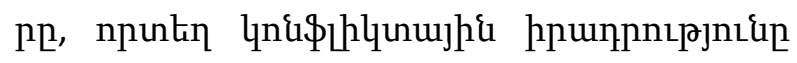

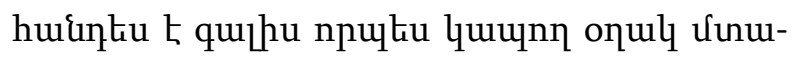

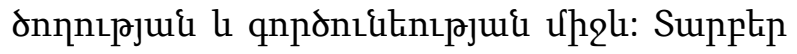

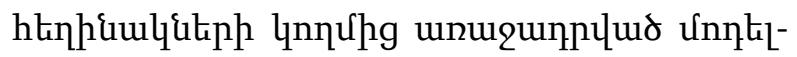

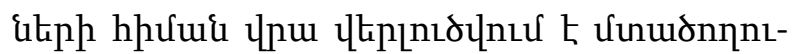

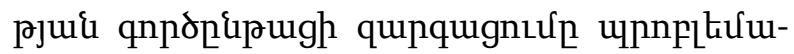

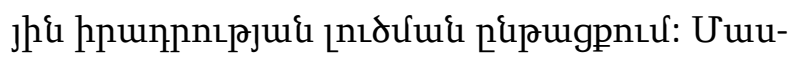

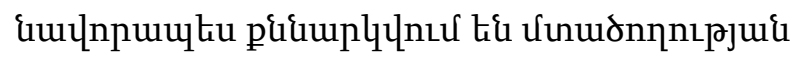

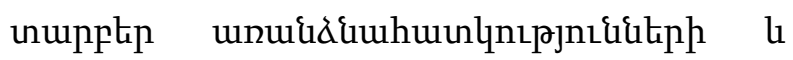

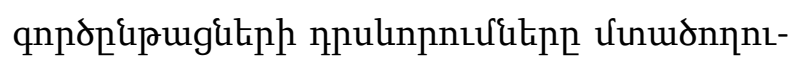

pjui qnponfapugh qunqugưui unupptip

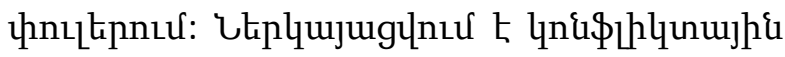

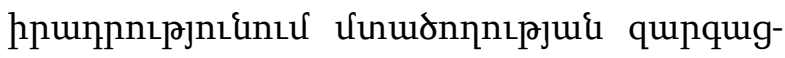

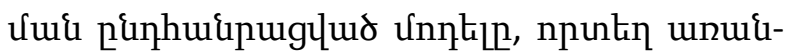

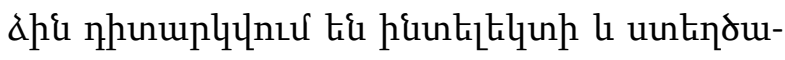

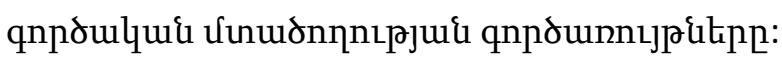

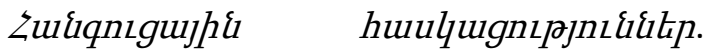

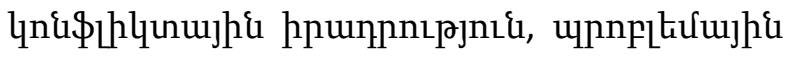

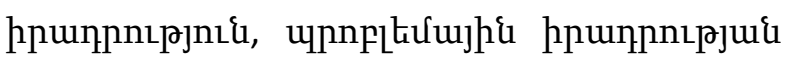

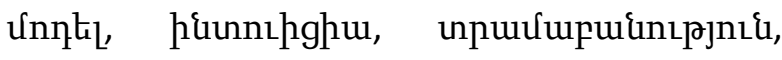

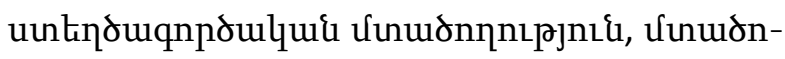

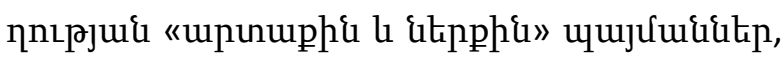

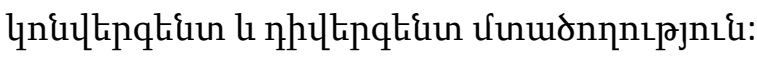

TEVOSYAN MARIA

\section{THE CREATIVE THINKING IN A CONFLICT SITUATION}

\section{SUMMARY}

The role of creative thinking in a conflict situation is analyzed in the article. The "internal and external" thinking conditions are presented where the conflict situation is acting as a linkage between thinking and activity. The development of thinking process during the settlement of a conflict situation is analyzed based on models suggested by different authors. Particularly, demonstrations of various peculiarities and processes of thinking in different stages of the thinking process development are discussed. The generalized model of thinking in a conflict situation is presented where the functions of intellect and creative thinking are observed separately.

Key words and expressions: conflict situation, problematic situation, model of the problem situation, intuition, logic, creative thinking, "internal and external" thinking conditions, convergent and divergent thinking. 